\title{
Nebivaolol effect in patients with chronic obstructive disease of the lungs
}

\author{
Holov G.A. MD, Abdijalilova S.I. MD, Ganiev U.Sh. MD, \\ Salohidinov Sh.N. MD \\ Republican Research Center of Emergency Medicine, Tashkent, Uzbekistan \\ Corresponding author: B.M Shakirov \\ Business address: 2 Nor Yakubov proezd 3, \\ Samarkand, Uzbekistan. \\ E- mail: baburshakirov@yahoo.com \\ Home tel.: 3662 (373208)
}

KEYWORDS: Chronic obstructive disease of the lungs, nebivaolol, treatment,

\begin{abstract}
Introduction: Chronic obstructive disease of the lungs (CODL) is one of the leading cases of morbidity and mortality all over the world. It is one of the causes which may form the basis of sudden death, it serves as disturbance of cardiac contractions rhythm. Aim of the research is to evaluate the effect of adrenoreceptor - nebivaolol by selective blocker $\beta 1$ on pulmonary hypertension degree in patients with chronic obstructive disease of the lungs. Material and methods: 60 patients with chronic obstructive disease of the lungs of III-IV stage in exacerbation phase were enrolled in the study. The patients were subdivided into 2 groups: group A for comparison (n-25) and group B for study $(n=35)$. The patients of both groups received standard therapy, directed to elimination of inflammatory process and improvement of bronchial permeability: antibiotics, broncholytics, mucolytics. The patients of group B were treated with nebivaolol in the dosage $10 \mathrm{mg} 24$ hours $(5 \mathrm{mg}$ dose 2 times 24 hours) for 14 days. Results: In absence of negative effect on hemodynamic indexes, electrophysiological parameters and sporometric data reveal changes in the rate of cardiac contractions to adequate values and significant increase of tolerance to physical exertion in 2 weeks after taking nebivaolol. Decrease of heart rate correlates with decrease of pulmonary hypertension. Conclusion: Taking into account the received findings nebivaolol can be recommended for treatment of patients with chronic obstructive disease of the lungs and pulmonary hypertension.
\end{abstract}

\section{INTRODUCTION}

Chronic obstructive disease of the lungs (CODL) is one of the leading cases of morbidity and mortality all over the world [1 - 4]. It is one of the causes which may form the basis of sudden death, it serves as disturbance of cardiac contractions rhythm.

Chronic obstructive pulmonary disease (CODL) is a growing global epidemic that is particularly important in developing countries. Morbidity and mortality from CODL will rise as population's age and mortality from cardiovascular and infectious diseases falls. In addition, CODL is associated with several other diseases, such as cardiovascular diseases, osteoporosis, diabetes, and metabolic syndrome, more commonly than expected by chance. These associations are greater than expected from common aetiological factors, such as smoking, suggesting that these comorbidities may be causally associated with the mechanisms of CODL. Systemic effects and comorbidities of CODL increase both the risks of hospitalisation and mortality and the costs, and are therefore a topic of increasing concern.

In administration of these preparations it may be difficult to control therapeutic dosage because overdosage also cases cardiotoxic effect, possible development of sinus tachycardia, premature atrial contractions, supraventricular tachycardia, fibrillation of atria and ventricular arrhythmia. 
The aim of the research is to evaluate the effect of adrenoreceptor - nebivaolol by selective blocker $\beta 1$ on pulmonary hypertension degree in patients with chronic obstructive disease of the lungs.

\section{PATIENTS AND METHODS}

60 patients with chronic obstructive disease of the lungs of III-IV (severe, extremely severe) stage in exacerbation phase were enrolled in the study.

The criteria of enrollment in the study:

1) Chronic obstructive disease of the lungs III-IV stage;

2) Roentgenographic signs of emphysema;

3) sinus tachicardia;

4) $\mathrm{PH}$ (higher than $25 \mathrm{~mm} / \mathrm{Hg}$ at rest);

5) chronic pulmonary heart;

The criteria of exclusion from the study:

1) chronic form of atrial fibrillation;

2) paroxysmal disturbances of the rhythm;

3) fever.

The patients of able bodied - age mainly males suffering from chronic obstructive disease of the lungs for about 10 years are enrolled in the study. The patients were subdivided into 2 groups: group A for comparison ( $n-25)$ and group B for study $(n=35)$. The patients of both groups received standard therapy, directed to elimination of inflammatory process and improvement of bronchial permeability: antibiotics (cephalosporines of the third generation), broncholytics (thiotropy bromide or its combination with $\beta 2$-agonist of long duration effect), mucolytics.

The patients of group B were treated with nebivaolol in the dosage $10 \mathrm{mg} 24$ hours $(5 \mathrm{mg}$ dose 2 times 24 hours) for 14 days. All patients were taken electrocardiography at rest and were determined HR on the 1 st and 14th days.

Echocardiography was performed (with the same time intervals) on Fillips NNV VISER CHD apparatus. Standard parameters of hemodynamics, final diastolic size (FDS) of the LV, final systolic size (FSS) of the LV, beat level (BL), fraction of discharge (FD) of the LV, left atrium (LA), right atrium (RA), thickness of the right ventricle wall (TRVW), average pressure above the pulmonary artery $(\mathrm{PpA})$, relation of $\mathrm{E} / \mathrm{A}$ rates of the $\mathrm{RV}$.

The function of external breathing was studied by bodyplethysmography technique with employment of Master Screen Body (Jaeger) apparatus, with this the level of forced expiration per second (VFEI), forced vital capacity of the lungs (FVCL), FVIL relations were studied in dynamics.

The patients carried out daily control of arterial pressure themselves using Digital automatic Blood Pressure Monitor M 4 (Omron) apparatus with HR registration. Tolerance to physical exertion was estimated according to 6-min. walkink test on the 1st, 7th and 14th days, enrichment of the arterial blood with oxygen $(\mathrm{Sa} 02)$ was determined with pulseoxymeter MD 300C with the same time intervals.

\section{RESULTS}

Clinical description of patients included in the study is presented in Table I. Hhe formed groups did not differ on age, sex, clinical features, initial HR (more than 80 beats per min), standard therapy and that is why they were regarded as identical. In comparison of initial clinic instrumental indexes in the studied groups there were not any statistically significant differences. In the majority of patients the level of arterial blood oxygenation was compensated. Respiratory rate (RR) in

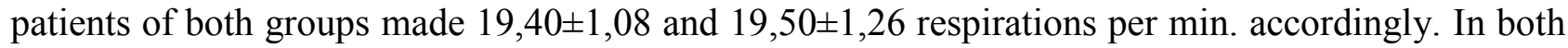
groups sinus tachycardia was registered, HR made $99,1 \pm 1,64$ beats per min in group A and 
$103,37 \pm 1,43$ beats per min. in group B. In the majority of patients the III functional class of chronic cardiac insufficiency (according to NYHA classification) is established according to 6-min. walking test results.

Table 1

Initial clinic instrumental description of patients with chronic obstructive disease of the lungs

\begin{tabular}{|l|l|l|l|}
\hline Index & Group for comparision (A) & Group for study (B) & $\mathrm{P}_{\mathrm{A}-\mathrm{-}}$ \\
\hline Age & $59,30 \pm 5,2$ & $61,30 \pm 4,3$ & $>0,05$ \\
\hline Males/females & $20 / 5$ & $28 / 7$ & $>0,05$ \\
\hline CODL duration & $8,90 \pm 3,9$ & $9,70 \pm 3,4$ & $>0,05$ \\
\hline ICD & 16 & 20 & $>0,05$ \\
\hline Hypertonic disease & 9 & 15 & $>0,05$ \\
\hline RR per/min & $19,40 \pm 1,08$ & $19,50 \pm 1,26$ & $>0,05$ \\
\hline FVCL \% & $64,20 \pm 4,46$ & $63,25 \pm 4,72$ & $>0,05$ \\
\hline VFEI \% & $41,97 \pm 3,60$ & $41,39 \pm 5,61$ & $>0,05$ \\
\hline VFEI/FVCL & $48,71 \pm 2,40$ & $50,23 \pm 5,66$ & $>0,05$ \\
\hline HR per/min & $99,10 \pm 1,64$ & $103,37 \pm 1,44$ & $>0,05$ \\
\hline PQ interval, mm & $0,14 \pm 0,003$ & $0,14 \pm 0,004$ & $>0,05$ \\
\hline QT interval, mm & $0,30 \pm 0,009$ & $0,31 \pm 0,011$ & $>0,05$ \\
\hline Sa0 ${ }_{2}, \%$ & $92,57 \pm 0,76$ & $90,59 \pm 0,63$ & $>0,05$ \\
\hline 6 min walking test & $226,11 \pm 16,68$ & $224,19 \pm 13,36$ & $>0,05$ \\
\hline
\end{tabular}

Note. CODL - Chronic obstructive disease of the lungs; IHD - ischemic heart disease; $\mathrm{RR}$ - respiratory rate; FVCL - forced vital capacity of the lungs; LFEI - level of forced expiration per a second; $\mathrm{HR}$ - heart rate; $\mathrm{SaO} 2$ - arterial blood oxygenation.

Initially in patients of both groups increase of thickness of RV wall was observed, it made $6,03 \pm 0,34 \mathrm{~mm}$ on the average, decrease of $\mathrm{E} / \mathrm{A}$ of RV correlation to $0,71 \pm 0,09$ and the level of average pressure over the pulmonary artery was increased to $38,0 \pm 2,8$ and $39,15 \pm 2,46 \mathrm{~mm} / \mathrm{Hg}$ accordingly (Table 2).

Table 2

Initial echocardiographic indexes in patients with for chronic obstructive disease of the lungs patients

\begin{tabular}{|c|c|c|c|}
\hline Parameter & Group comparision (A) $\mathrm{n}=25$ & Group for study (B) $\mathrm{n}=35$ & $\mathrm{P}_{\text {А-Б }}$ \\
\hline RA, mm & $39,88 \pm 1,17$ & $40,0 \pm 1,35$ & $>0,05$ \\
\hline TW RV, mm & $6,0 \pm 0,35$ & $6,03 \pm 0,35$ & $>0,05$ \\
\hline FDS RV, mm & $34,71 \pm 2,15$ & $35,32 \pm 1,66$ & $>0,05$ \\
\hline Ppa, mm. Hg & $38,0 \pm 2,8$ & $39,15 \pm 2,46$ & $>0,05$ \\
\hline E/A (RW) & $0,71 \pm 0,09$ & $0,72 \pm 0,07$ & $>0,05$ \\
\hline
\end{tabular}

Note. In table 2 : CODL- chronic obstructive disease of the lungs; RA - right atrium; TW - thickness of the wall; RV - right ventricle; FDS - final diastolic size; Ppa — pressure over pulmonary artery. 
In patients of the studied group (group B) good tolerance of nebivaolol was noted. During the whole period of treatment with this preparation side effects were not registered in any of cases, noted worsening of cough, edema, and development of respiratory discomfort. In the group of patients receiving nebivaolol during two weeks statistically significant increase of E/A RV correlation was noted $-81 \pm 0,08$ and $0,72 \pm 0,08(\mathrm{p}<0,05)$, compared to the patients who did not receive neivaolol, but it did not achieve the norm (normal correlation in persons older than 50 years makes $1,34 \pm 0,4$ ). LV FD on the background of standard therapy and in taking nebivolol did not change (table 3 ). In two weeks after performed therapy in patients of both groups tolerance to physical exertion increased.

On the background of standard therapy (group A) the distance covered in 6 min. walking test increased from $226,11 \pm 16,68$ to $261,42 \pm 39,58 \mathrm{~m}(\mathrm{p}<0,05)$. When nebivaolol is added to standard treatment (group B) statistically more significant increase of the covered distance from $224,19 \pm 13,36$ to $296,6 \pm 68,02 \mathrm{~m}(\mathrm{p}<0,001)$ was noted.

Table 3

Dynamics of echocardiographic parameters on the background of standard treatment and in administration of nebivaolol in chronic obstructive disease of the lungs

\begin{tabular}{|c|c|c|c|c|}
\hline \multirow{2}{*}{ Parameter } & \multicolumn{2}{|l|}{ Group A } & \multicolumn{2}{|l|}{ Group B } \\
\hline & initially & In 2 weeks & initially & In 2 weeks \\
\hline FDS LV, mm & $45,64 \pm 0,85$ & $45,64 \pm 0,85$ & $47,42 \pm 1,02$ & $46,78 \pm 0,91$ \\
\hline FSS LV, mm & $29,87 \pm 0,83$ & $29,94 \pm 0,78$ & $31,22 \pm 1,07$ & $30,52 \pm 0,84$ \\
\hline FDV LV, ml & $96,41 \pm 4,19$ & $96,41 \pm 4,19$ & $102,25 \pm 3,53$ & $101,78 \pm 4,5$ \\
\hline FSV LV, ml & $35,29 \pm 2,1$ & $35,29 \pm 2,14$ & $40,17 \pm 3,98$ & $37,52 \pm 2,33$ \\
\hline PL, ml & $61,11 \pm 2,31$ & $61,13 \pm 2,35$ & $66,03 \pm 2,45$ & $64,52 \pm 2,69$ \\
\hline $\mathrm{FD}, \%$ & $63,64 \pm 1,02$ & $63,64 \pm 1,02$ & $62,35 \pm 1,61$ & $62,00 \pm 1,19$ \\
\hline $\mathrm{LA}, \mathrm{mm}$ & $36,23 \pm 0,88$ & $36,41 \pm 0,88$ & $37,57 \pm 1,29$ & $36,84 \pm 1,81$ \\
\hline $\mathrm{RA}, \mathrm{mm}$ & $39,88 \pm 1,17$ & $39,0 \pm 1,26$ & $40,0 \pm 1,35$ & $41,05 \pm 1,21$ \\
\hline TW RV, mm & $6,0 \pm 0,35$ & $5,79 \pm 0,27$ & $6,03 \pm 0,34$ & $6,41 \pm 0,39$ \\
\hline FDS RV, mm & $34,71 \pm 2,15$ & $36,93 \pm 2,0$ & $35,32 \pm 1,66$ & $35,05 \pm 1,83$ \\
\hline E/A RV & $0,71 \pm 0,09$ & $0,70 \pm 0,07$ & $0,72 \pm 0,09$ & $0,81 \pm 0,08 * *$ \\
\hline
\end{tabular}

Note. *- $\mathrm{p}<0,05 * *-\mathrm{p}<0,001$ in comprasion with initial level. LV - left ventricule; FSS -final systolic size; FDV - final diastolic level; FSL- final systolic level; PL - pressing level; FD - fraction of discharge; LA - left atrium.

The best results in group B were probably received due to HR decrease. In patients of both groups (Fig. 1) statistically significant HR decrease was registered $(\mathrm{p}<0,05)$, although HR decrease to normal (less than 70 beats per min.) was only achieved in taking nebivaolol. HR decrease in $92 \%$ of patients was already noted from the $2 \mathrm{~d}$ day of taking the preparation but statistically significant HR decrease was observed in 2 weeks from beginning of treatment $(p<0,05)$.

PH (pulmonary hypertension) is one of the most important factors of prognosispatients with in cronic obstructive disease of the lungs. In the internist,s practice use of blockers of calcium channels, prazozine, nitrates is limited for these patients due to side effects and bozentane therapy is recommended at present only for treatment of idiopathic $\mathrm{PH}$ and $\mathrm{PH}$ in scleroderma. In our research standard treatment did not effect $\mathrm{PH}$ degree $(\mathrm{p}>0,05)$, average pressure above pulmonary artery statistically decreased significantly in the group of patients taking nebivaolol from $15 \pm 2,46$ to $36,66 \pm 3,73$ mm/Hg. (p<0, 05; Fig. 2). 

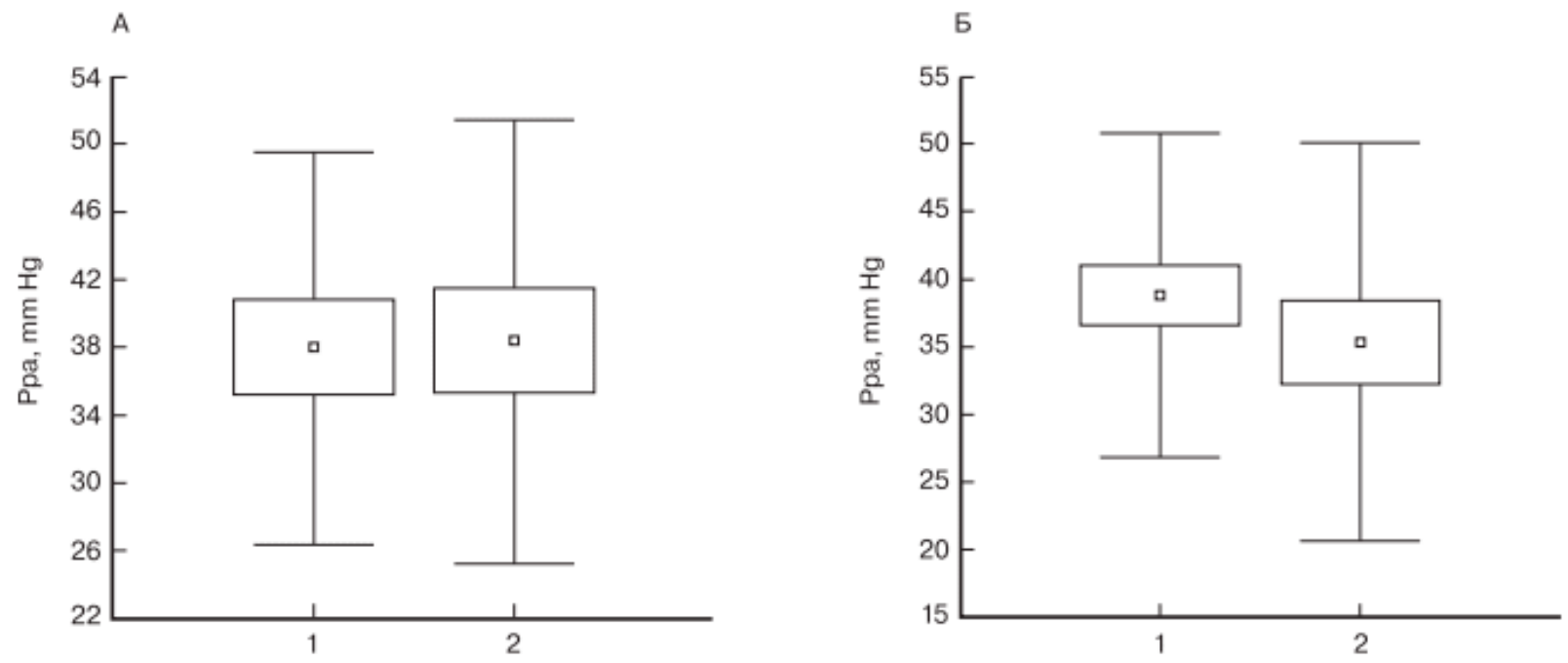

工 \pm Std. Dev. $\square \pm$ S td. Err. a Mean

Fig. 1, 2. Dynamics of average pressure in pulmonary artery (Ppa) in patients with chronic obstructive disease of the lungs on the background of standard therapy (A) and in administration of nebivolol (B).

In determination of correlation between HR and average pressure over the pulmonary artery changes, direct negative connection is revealed: decrease of HR and average pressure over the pulmonary artery on the background of nebivolol treatment. The chart of the objects of investigation disperse in coordinates adequate to two studied signs clearly illustrates connection between parameters (Fig.3).

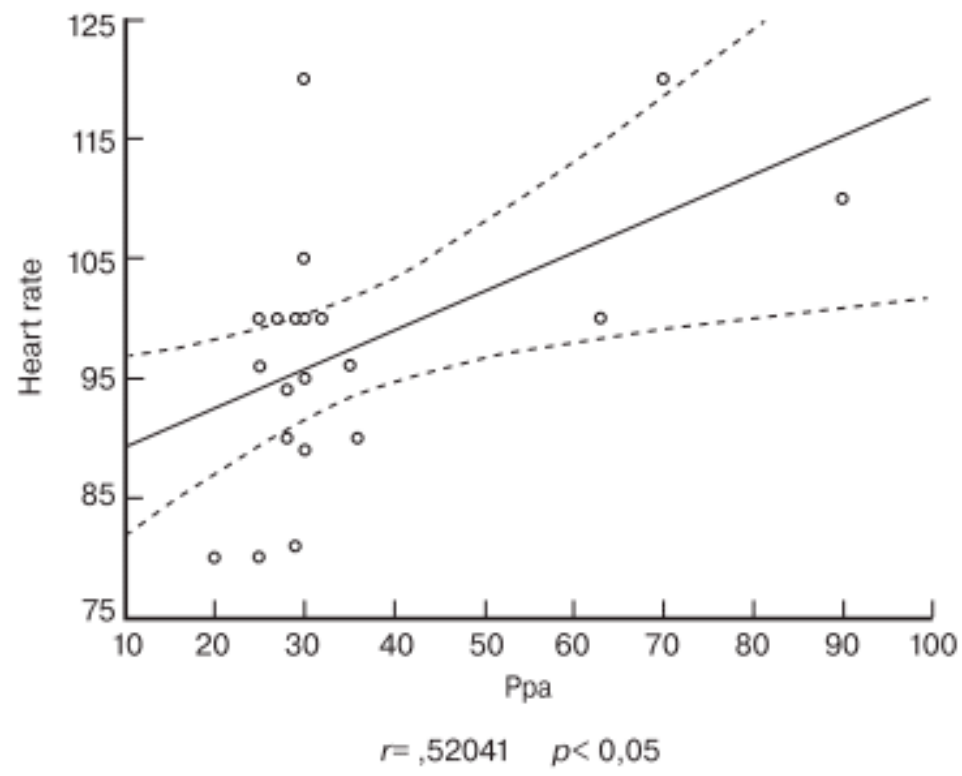

Fig 3. Correlation between average pressure over the pulmonary artery (Ppa) and HR in patients with chronic obstructive disease of the lungs on the background of nebivolol treatment. Ppa - average pressure in pulmonary artery $\mathbf{m m} / \mathrm{Hg}$.

Change of hemodynamic in pulmonary circulation probably contributed to clinical improvement of condition of patients with chronic obstructive disease of the lungs according to 6min walking test findings. HR decrease influences the risk of hospitalization and death for cardiovascular diseases, thus use of nebivolol makes it possible to improve therapy in patients with chronic obstructive disease of the lungs of severe stage and $\mathrm{PH}$. 


\section{DISCUSSION}

Among the factors causing the development of arrhythmia are considered to be the following: aggravation of bronchial permeability, medicines prescribed to patients with chronic obstructive disease of the lungs, dysfunction of autonomic conductive cardiac system, ischemic heart disease, arterial hypertonia, dysfunction of the left ventricle (LV) and right ventricle (RV), increase of catecholamine level in the blood and development of hypoxemia. In severe course of chronic obstructive disease of the lungs the development myocardiodystrophy, intensifies contractive inadequacy of the myocardium even in absence of coronary disease that may be accompanied by pains in the heart, arrhythmia [5 - 7].

Anticholenergetic preparations, $\beta 2$-agonists and xanthines are used as bronchodilatators in recent years [8-9].

Introduction of nebivaolol into the scheme of treatment of patients with severe and extremely severe chronic obstructive disease of the lungs is statistically important in decrease of pulmonary hypertension degree.

In absence of negative effect on hemodynamic indexes, electrophysiological parameters and sporometric data reveal changes in the rate of cardiac contractions to adequate values and significant increase of tolerance to physical exertion in 2 weeks after taking nebivaolol.

\section{CONCLUSION}

Morbidity and mortality from CODL will rise as population's age and mortality from cardiovascular and infectious diseases falls.

Decrease of heart rate correlates with decrease of pulmonary hypertension in patients with chronic obstructive disease of the lungs of III-IV degree.

In absence of negative effect on hemodynamic indexes, electrophysiological parameters and sporometric data reveal changes in the rate of cardiac contractions to adequate values and significant increase of tolerance to physical exertion in 2 weeks after taking nebivaolol.

Taking into account the received findings nebivaolol can be recommended for treatment of patients with chronic obstructive disease of the lungs and pulmonary hypertension.

Acknowledgements

We wish thank Shakirov B.M. for their help with project and the preparation of the manuscript.

\section{REFERENCES}

[1]. Adamyan K.G., Chilingaryan A.L. Comparative effectiveness of ivabradine and verapamil in patients with diastolic cardiac insufficiency due to impaired relaxation of the left ventricle. Rational Pharmacotherapy in cardiology; 2009:3:31-35.

[2]. Singch B.N. Impact of reduced heart rate. Morbidity in cardiovascular disorders. J. Cardiovasc Pharmacol Ther.2001; 6:313-31.

[3]. Reil J.C., Bohm M. Beautiful results the slower, the better? Lancet 2008; 372:779-80.

[4]. Fox K., Ford I., Steg P.G., Tendera M, Robertson M., Ferrari R. Heart rate as a prognostic risk factor in patients with coronary artery disease and left-ventricular systolic dysfunction (Beautiful): a subgroup analysis of a randomized controlled trial. Lancet 2008; 372:817-821.

[5]. Salpeter S.R., Ormiston T.M., Salpeter E.E. Cardiovascular effects of beta-agonists in patients with asthma and COPD: a meta-analysis. Chest 2004; 125:21-3.

[6]. Thollon C., Cambarrat C., Vian J., Prost J.F., Peglion J.L., Vilaine J.P. Electrophysiological effects of 16257, a novel sino-atrial node modulator, in rabbit and guinea- pig cardiac preparations: comparison with UL-FS 49. Br J Pharmacol 1994; 112:37-42. 
[7]. Fomin I.V., Valiculova F.Yu. Antianginal effect of long use of avabradine in patients with ischemic heart disease and diabetes mellitus. Journal Cardiology 2009; 3:9-5.

[8]. Komlev A.D., Volkova A.L., Arutunov V.A. Influence of canals blocker Ifivabrodine on the indexes of external breathing functions in patients with chronic obstructive disease of the lungs in the period of stable course of the disease. Ter. Arch. 2010; 3:23-7.

[9]. Abdurarachmanova G.M., Zulkarneev R.H., Zagudulin N.S. Effect of Ifvabradine and salbutamol combination on heart rate frequency and external breathing function in patients with chronic obstructive disease of the lungs in combination with IHD. Collection of thesis of XX congress on respiratory organs disease; 10-11. 